\title{
Constats sur la gouvernance des territoires
}

\author{
Marc-Urbain Proulx \\ CRDT-UQAC
}

\section{INTRODUCTION}

Dans sa version contemporaine, la réforme territoriale fut lancée au Québec dans les années 1960. Plusieurs études ${ }^{1}$ inspirées par les nouvelles sciences urbaines, régionales, rurales, métropolitaines, illustraient à l'époque de nombreux problèmes sur les divers territoires qui composent la nation. Le sentiment largement répandu pointait un nécessaire coup de barre en matière d'organisation générale de l'espace ${ }^{2}$. Grâce aux nouveaux transferts fédéraux dans le champ local et régional de compétence provinciale, le gouvernement du Québec a créé l'OPDQ (Office de planification et de développement du Québec) en 1968 pour éclairer la situation et intervenir judicieusement. Du coup, il reconnaissait la nécessité de bien articuler la nouvelle gouvernance territoriale à instaurer. Trois dimensions principales se sont affirmées au réformateur, soit la gestion publique de programmes, de biens et de services près de la population, l'encadrement de l'aménagement ainsi que le

\section{AUTORITÉS LOCALES}

En 1961, quelque 1748 municipalités fortement diversifiées et près de 2000 commissions scolaires découpaient finement l'espace québécois en multiples territoires locaux. Ce qui offrait au total énormément d'élus par tranche de population, notamment dans les zones peu populeuses. Les bassins de clientèles étaient très souvent restreints ${ }^{3}$ puisque plus de $80 \%$ des municipalités dénombraient moins de 3000 habitants. Plusieurs biens et services typiquement collectifs n'étaient pas assumés soutien au développement économique, social et culturel.

\section{Cet article propose quelques jalons de base d'un constat général sur la gouvernance des territoires au Québec.}

Depuis ce temps, la réforme québécoise chemine par étapes distinctes, plus ou moins progressives, dont celle vécue actuellement avec les mesures gouvernementales de 2014-15 que d'aucun associe à l'austérité. Viendront surement d'autres étapes. Or, cinq décennies après le coup d'envoi, nous ne disposons d'aucune étude exhaustive sur le chemin parcouru. Ce texte ne prétend évidemment pas à cette exhaustivité aussi nécessaire soit-elle pour offrir de la perspective. Il propose plus modestement quelques jalons de base d'un constat général sur la gouvernance des territoires au Québec.

par le secteur public. Les disparités et les resquillages dans les services municipaux étaient nombreux. Peu de villes disposaient d'un plan d'urbanisme. Le désordre dans l'usage du sol était considérable en imposant de nombreuses incompatibilités, iniquités et incohérences. L'érosion de la population dévastait de nombreuses municipalités rurales. L'impôt foncier illustrait ses limites. Les initiatives locales novatrices étaient beaucoup trop rares. 
Figure 1 - Évolution du régime municipal au Québec

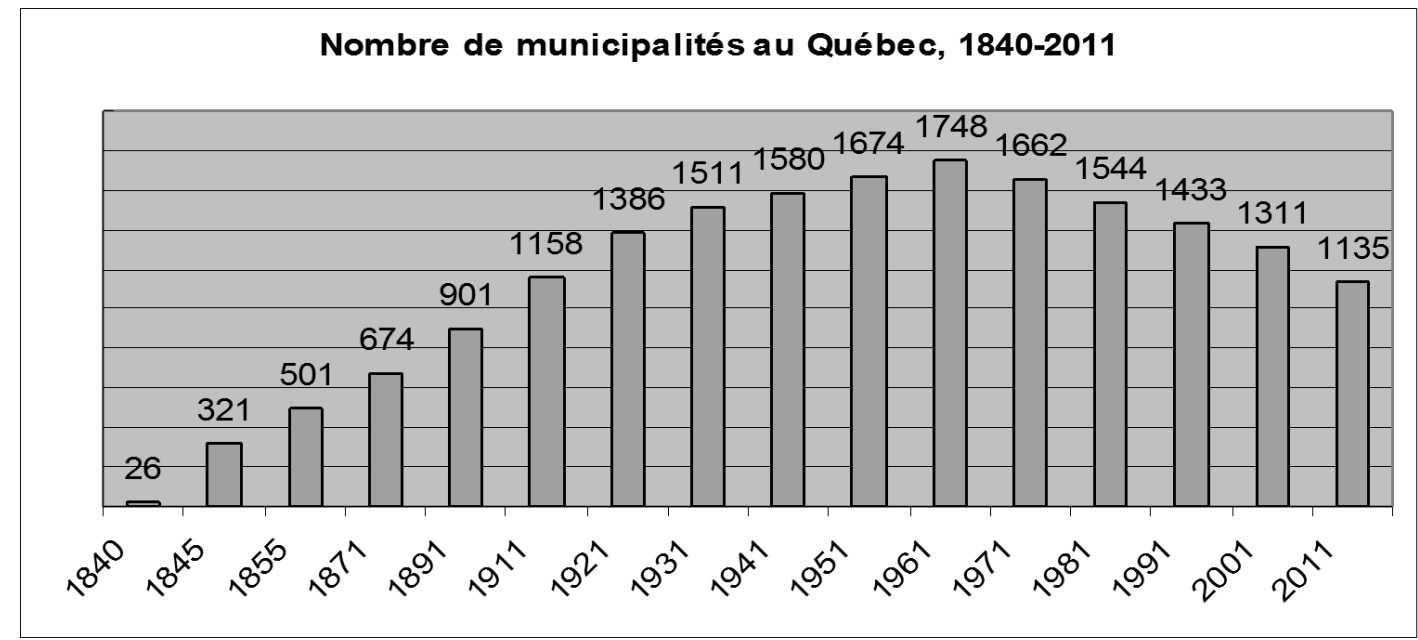

Source : Proulx, M.U. « Territoires et développement : la richesse du Québec », PUQ, 2011

On illustra par ailleurs la présence de nombreuses municipalités dont la localisation avantageuse occasionne la polarisation plus ou moins forte des activités privées et publiques. Un système dynamique de pôles ${ }^{4}$ fut établi comme logique pour ordonnancer les interventions publiques. Certains s'inscrivent tels de véritables pôles ruraux de développement dont les effets structurants bénéficient à leur zone de rayonnement ${ }^{5}$. D'autres pôles, centraux sur leur territoire, ont intérêt à maximiser l'organisation de celui-ci pour nourrir les forces centripètes dont ils profitent. Bien positionnés dans la hiérarchie urbaine, d'autres pôles encore ont bénéficié en 2001 de la fusion de leur agglomération et souhaitent de nouvelles mesures consolidatrices afin de mieux jouer leur rôle moteur sur leur espace de rayonnement. Parmi ceux-ci, les avant-postes en périphéries, comme Sept-Îles, Val-d'Or, Saguenay, Rivière-du-Loup, illustrent des potentiels géoéconomiques considérables. Tandis que déclassée par Toronto au Canada, la métropole Montréal cherche à se repositionner dans le réseau mondial des «Global Cities-Regions » ${ }^{6}$ en misant notamment sur sa périphérie désormais rétrécie. Quelle que soit la taille des pôles québécois, le principe de leur renforcement fut bien accueilli comme stratégie québécoise. Restait à leur trouver des foyers de croissance et de développement.

D’une manière générale, Québec désirait accentuer la prise en main locale de responsabilités publiques et de leviers de développement. Pour ce faire, il a beaucoup misé sur les élus municipaux. Québec a aussi fait appel à la démocratie participative, à la concertation élargie et aux ententes intermunicipales. Des fonctions publiques furent décentralisées ${ }^{7}$, à certains degrés. Québec a aussi bonifié considérablement son traditionnel encadrement des fonctions locales avec des normes, des incitatifs, des financements conditionnels. En réalité, Québec conserva la main haute. Le nombre de Commissions scolaires diminua radicalement pour atteindre 72 actuellement. Plusieurs fusions et regroupements municipaux eurent lieu au fil des décrets gouvernementaux généralement douloureux. Ainsi, le nombre total de corporations municipales fut réduit du tiers (figure 1). Néanmoins, le Québec municipal demeure toujours très morcelé, certes trois fois moins que la Saskatchewan, mais beaucoup plus que l'Ontario et la Colombie Britannique $^{8}$. En conséquence, encore 700 à 800 municipalités offrent une base fiscale trop limitée pour exercer convenablement des fonctions publiques désormais techniquement complexes qui nécessitent d'importantes masses critiques de clients. Aussi, le nombre d'élus locaux reste très élevé, tandis que leur renouvèlement s'avère problématique9. Malgré les incontestables avancées, la question de l'efficacité globale du secteur public local conserve encore sa grande pertinence.

Plusieurs fusions et regroupements municipaux eurent lieu au fil des décrets gouvernementaux généralement douloureux. 


\section{RÉGIONS ADMINISTRATIVES}

L'administration publique québécoise déconcentrée possède déjà une très longue feuille de route. $\mathrm{Au}$ début des années 1960, on dénombrait autour de 1000 bureaux locaux, près de 400 bureaux régionaux et plus de 65 aires différentes pour appliquer les déjà nombreuses opérations régionalisées des différents ministères et organismes ${ }^{10}$. Comme solution à cet éclatement, des aires uniformes furent découpées en 1966, en épousant largement le rayonnement des principaux pôles urbains ${ }^{11}$.

\section{Le territoire commun d'interventions rend en principe possible la création de complé- mentarités, de convergences et de synergies.}

Ainsi sont apparues les dix premières régions administratives comme assise de la coordination et de la cohérence en matière de gouvernance territoriale (gestion - aménagement - dévelop- pement). Le territoire commun d'interventions rend en principe possible la création de complémentarités, de convergences et de synergies. À cet effet, la planification régionale s'est tout de suite inscrite telle une mission incontournable ${ }^{12}$. Pour ce faire, différents mécanismes de médiation entre les acteurs furent expérimentés avec plus ou moins de bonheur au fil du temps, notamment les CRD (conseils régionaux de développement), les délégations régionales de l'OPDQ, les CAR (conférences administratives régionales), les ministres régionaux, les députés régionaux délégués du premier ministre, les sous-ministres-adjoints en régions. À cet effet, la LAOVT (loi pour assurer l'occupation et la vitalité des territoires) de 2012 préconise le renforcement des CAR, sans spécifier comment. La question demeure ouverte. Dans un esprit de réponse, le démantèlement des CRÉ (conférences régionales des élus) en 2014-15 ne surprend guère ceux et celles qui effectuent une lecture chronologique de la splendeur et du déclin de ces organismes.

Figure 2 - Construction institutionnelle des régions 2014

\begin{tabular}{llll} 
Découpage initial : + 80 \% & Décrets 1987:20 à 40\% & & Sans statut régional \\
\cline { 1 - 1 } Outaouais & Laval & Beauce \\
Saguenay-Lac-Saint-Jean & Chaudière-Appalaches & Charlevoix \\
Mauricie & Bas-Saint-Laurent & Kamouraska \\
Estrie & Centre-du-Québec & Témiscamingue \\
Gaspésie-Îles & Nord-du-Québec & Lac-Saint-Jean \\
Québec & Lanaudière & Richelieu \\
Montréal & Laurentides & Basse-Côte \\
Abitibi-Témiscamingue & & Baie-des-Chaleurs \\
Côte-Nord & & \\
Montérégie & &
\end{tabular}

Source : Compilations du CRDT - UQAC à partir d'enquêtes et archives

Dans les années 1970 et 1980, les ancêtres des CRÉ, soit les CRD, ont effectué un splendide travail en matière de coordination des actions vers des objectifs globaux pour les régions administratives ${ }^{13}$. La planification se voulait globale avec des moyens importants pour documenter substantiellement le profil de chaque région et pour évaluer les options. Des consensus autour de priorités furent scellés grâce à la concertation réussie entre les acteurs territorialisés. Des actions collectives structurantes ont été élaborées et exécutées, souvent en suscitant des synergies régionales. 
L'une des réussites à cet effet s'est inscrite dans la mise en ouvre de conseils régionaux sectoriels dans la culture, l'environnement, le tourisme, l'éducation supérieure, etc. qui agissent notamment comme une « oreille du milieu » auprès de leur direction régionale ministérielle.

L'une des réussites à cet effet s'est inscrite dans la mise en œuvre de conseils régionaux sectoriels dans la culture, l'environnement, le tourisme, l'éducation supérieure, etc. qui agissent notamment comme une « oreille du milieu » auprès de leur direction régionale ministérielle. Aussi, de 1983 à 1991, deux générations d'une nouvelle démarche originale de planification interactive et mobilisatrice dite «Sommet» furent expérimentées. Elle a été comparativement plus innovatrice que les presses dentent sous l'angle du nombre et surtout de l'originalité des actions proposées ${ }^{14}$. En réalité lors de ces Conférences socioéconomiques, un très bon équilibre fut atteint entre les quatre dimensions de la planification territoriale (vision - cadre - faisabilité décisionnelle - interaction) facilitant ainsi l'émergence d'initiatives innovatrices ${ }^{15}$. À cette époque, le régionalisme instrumentalisée a atteint son zénith, avec à la clé de fortes revendications politiques. Un moratoire de Québec fut imposé en 1991 à cette forme de planification régionale.

Une importante lacune ombre cette période effervescente. Elle réside dans le peu de véritables leviers de développement régional qui furent appropriés par les institutions vigoureusement mises à l'œuvre. Soulignons d'abord que malgré l'utilisation de concepts pertinents comme la RLDD (région laboratoire de développement durable), aucune région n'a pu conduire sa réflexion collective jusqu'à l'élaboration d'un véritable projet de collectivité pouvant maximiser les convergences

\section{RÉGIONALISME REFROIDI}

Le mouvement régional a subi un virage important au tournant des années 1990. Plusieurs facteurs y ont contribué. Le déclencheur réel fut le retrait du gouvernement fédéral de ses transferts financiers ciblés sur cette mission régionale au Québec. Mission qu'il exerce désormais luimême avec Développement économique Canada entre les multiples actions corporatives. Aucune région non plus ne s'est dotée d'un concept d'aménagement capable de proposer les grandes orientations et les grands choix en matière d'équipements et d'infrastructures. Sous l'angle du développement, les stratégies spécifiquement régionales furent rares.

Une importante lacune qui ombre cette période effervescente réside dans le peu de véritables leviers de développement régional qui furent appropriés par les institutions vigoureusement mises à l'œuvre.

Si des fonds de développement régional ciblés pour le financement d'initiatives ont été régulièrement mis en place par Québec depuis 1975, aucun n'a pu émerger de l'épargne locale et régionale dans un esprit de cumul territorial de capitaux pour investir. Il n'y a pas au Québec de société publique régionale d'investissement dans le style du portefeuille CREECO chez la nation Crie. Déjà expérimenté par plusieurs villes y compris Montréal International, aucune région ne s'est dotée d'un service professionnel de prospection d'activités économiques. On dénombre que très peu d'actifs régionaux et aussi très peu de services spécialisés desservis à cette échelle. Bref, l'apprentissage collectif a certes progressé au sein de la nouvelle élite régionale, mais dans des limites très évidentes sous l'angle de l'appropriation de leviers. En matière de développement en réalité, les régions n'ont pas trouvé leur niche entre le fort localisme des élus municipaux et l'État québécois à l'affut de nouveaux outils nationaux tels qu'Investissement Québec, Hydro-Québec, Fonds de solidarité, Innovatech, etc.

(DEC). Précisons que les nouvelles agences régionales mises en place par DEC n'ont pas mis à profit la formule de démocratie participative déjà bien rodée en régions à cette époque. En perdant sa contribution financière fédérale, l'OPDQ fut démantelé. Québec a aussi ralenti son soutien au développement régional. À titre 
d'exemple, les nouvelles régions créées par décret en 1987 n'ont atteint que $38 \%$ de leur objectif en matière de mise en place d'agences sectorielles ${ }^{16}$. Tandis que d'autres régions naturelles et éligibles sont demeurées sans statut (figure 2).

\section{Du côté économique, les grappes industrielles sont devenues le fer de lance de la promotion du développement par Québec.}

Dans la foulée, le gouvernement du Québec a décidé de miser sur une approche sectorielle à l'échelle régionale. Fut à cet effet doublement adoptée ladite planification stratégique. Du côté économique, les grappes industrielles sont devenues le fer de lance de la promotion du développement par Québec. Et ce, malgré le fait qu'il n'y a pas a priori de grappes industrielles véritablement territorialisées au Québec ${ }^{17}$. Chaque région devait néanmoins faire un $1^{\text {er }}$ choix de spécialisation économique appelée « créneau d'excellence » à construire in situ. Des agences de promotion de ces spécialisations régionales furent établies, notamment dans les secteurs de l'agroalimentaire, du multimédia, de l'aluminium, du maritime, de l'optique photonique, des mines, etc. Des analyses de champs concurrentiels et de filières de production ont permis de cibler des occasions de valeur ajoutée. La magie du «clustering» devait en principe s'opérer par les « spill-over-effects $^{18}$ » stimulés par la culture entrepreneuriale, les allègements fiscaux, les transferts technologiques, le réseautage, etc.

Du côté administratif, les secteurs publics régionalisés comme la santé, l'éducation, la culture, etc. doivent chacun aussi, désormais, effectuer une lecture stratégique afin d'améliorer l'efficacité de leur fonctionnement interne. Cette pratique a conduit notamment à instituer les ententes spécifiques de gestion devenues une formule généralisée pour allouer les ressources des programmes gouvernementaux en fonction des préférences locales et régionales.
Bien qu'aucune évaluation ne soit disponible, ce virage sectoriel en matière de planification a certes eu comme effet de placer en seconde priorité non seulement la globalité du visionnement régional et le système de relations intersectorielles, mais aussi l'enjeu de la diversification de la structure économique des régions. Pourtant, les succès régionaux comme ceux de la Beauce, des Bois-Francs, des Laurentides, de l'Estrie et de plus en plus du BasSaint-Laurent illustrent justement les vertus de la diversification économique.

Bref, il est compréhensible que l'approche globale traditionnelle des CRD soit devenue de plus en plus difficile à exercer. D'autant plus que le soutien territorial aux initiatives et à l'entrepreneuriat fut confié aux nouveaux CLD de 1998. Transformés en CRÉ en 2003 par la réduction considérable du pluralisme de représentation à son Conseil afin de donner préséance aux élus locaux, ces organismes ont clairement subi un affaiblissement de leur rôle de concertation. Leur planification fut de plus en plus restreinte à une simple sommation des stratégies et des actions corporatives portées par leur milieu. Signalons aussi que l'élimination en 2003 de leur Association québécoise fut une lourde perte en matière de réflexivité. En conséquence, les besoins et les idées véhiculées vers la Table Québec - Régions (TQR) se sont banalisés et éclatés tous azimuts. Dans le flou ambiant, les CRÉ ne semblaient pas voir « l'épée de Damoclès ». À un point tel que les propositions de renouvèlement formulées par le CÉRO en Outaouais, par le Forum de la société civile en Mauricie et par Vision 2025 au Saguenay-Lac-Saint-Jean ont provoqué la suspicion plutôt que le réveil. En 2012, la LAOVT précitée ne mentionnait aucunement ce que deviendraient les CRÉ. On le devinait.

Les succès régionaux comme ceux de la Beauce, des Bois-Francs, des Laurentides, de l'Estrie et de plus en plus du Bas-SaintLaurent illustrent justement les vertus de la diversification économique. 


\section{APPROCHE DESCENDANTE}

Ce geste gouvernemental qui élimine actuellement les CRÉ frappe néanmoins l'imaginaire. Plus fondamentalement, la politique territoriale illustre depuis quelques années une approche plus dirigiste. Plutôt que de déléguer largement vers les territoires, Québec relève lui-même certains enjeux stratégiques. Ce fut le cas avec la périphérie nord que le Conseil exécutif a décidé de planifier en effectuant même un découpage territorial spécial statistiquement non saisissable $^{19}$. Les grandes régions de Montréal et de Québec furent aussi l'objet d'une initiative planificatrice de Québec (plans métropolitains d'aménagement et développement) afin de concilier les multiples roitelets autour des problèmes réels, notamment l'étalement urbain et le transport collectif. Face à la forêt boréale, Québec a réagi pour interpeler les enjeux complexes de ce secteur d'activité en changements rapides ${ }^{20}$. Fut organisé à cet effet un Rendez-vous national de la forêt québécoise en 2013 dont le suivi actuel nécessite largement les interventions de Québec et des acteurs du terrain. Cette nouvelle approche dirigiste fut aussi bien visible récemment au Saguenay_Lac-Saint-Jean avec l'organisation par Québec, en juin 2015, d'un sommet économique simplement consultatif des élites locales et régionales. L'approche descendante ou «Top Down» pour relever les enjeux territorialisés se poursuit actuellement avec la stratégie maritime. Elle se poursuivra sans

\section{PROGRÈS COMMUNAUTAIRE}

En effet, depuis plus de trois décennies, les assises supralocales MRC progressent certes lentement, mais néanmoins surement en matière d'organisation territoriale de fonctions publiques qui nécessitent à la fois la proximité de la population, une masse critique de clients et l'engagement des élus locaux responsables. On assiste en réalité à une véritable construction institutionnelle territoriale par diverses petites organisations monofonctionnelles à travers lesquelles on retrouve les corporations doute dans le futur rapproché avec les zones pertinentes telles que les couronnes périurbaines ciselées par l'urbanisation diffuse, les poches dévitalisées formées par les municipalités en érosion de leur population, la fosse minérale du Labrador, l'aire métropolitaine OttawaGatineau, l'anneau sud-est composé de pôles prospères ainsi que les corridors en densification de la Beauce, des Laurentides, de la BasseMauricie, du Saguenay (Alma - La Baie), du Basdu-Fleuve.

\section{Les initiatives de Québec sont appréciées sur ces territoires émergents qui ne respectent souvent pas les frontières des découpages institutionnalisés}

En réalité, des enjeux territoriaux affirment leur caractère stratégique. Les initiatives de Québec sont appréciées sur ces territoires émergents qui ne respectent souvent pas les frontières des découpages institutionnalisés ${ }^{21}$. Ce qui ne doit pas faire oublier cependant que plusieurs autres enjeux sont déjà interpelés ou en voie de l'être à l'échelle communautaire des opérateurs ancrés dans les collectivités territoriales. Le principe de subsidiarité s'inscrit en force, en représentant un atout dont le Québec ne peut se passer. Voyons un peu.

MRC de nature multifonctionnelles. Construction qui est toujours en cours, notamment dans de nouveaux champs pertinents pour l'action comme le patrimoine, l'agriculture, l'environnement, la ruralité, les transferts technologiques, les paysages, l'incubation d'entreprises, le transport collectif, la forêt. Signalons que plusieurs territoires MRC furent redessinés en 2001 avec la fusion des 14 agglomérations urbaines qui s'ajoutent désormais au nombre total. 


\section{Figure 3 - Construction institutionnelle des territoires MRC 2014}

Fortement (+66 \%)
Aménagement
Travail / emploi
Soutien aux entreprises
Santé, services sociaux
Financement des initiatives
Jeunesse-Emploi
Gestion des déchets
Évaluation foncière

Moyennement (33 à $66 \%$ )
Promotion industrielle
Environnement
Scolaire
Commerce
Tourisme
Agriculture
Forêts
Prospection de projets

\author{
Faiblement (-33\%) \\ Éducation supérieure \\ Culture \\ Innovation \\ Banque de terrains \\ Sports et loisirs \\ Voirie tertiaire \\ Villégiature \\ Patrimoine
}

Source : Compilations du CRDT - UQAC à partir d'enquêtes et archives

Toutes ces fonctions publiques de la figure 3 s'exercent autour de quatre grandes missions. D'abord, les Conseils MRC sont responsables des orientations en matière d'aménagement du territoire. Leurs aires de gouvernance servent aussi d'assises pour la gestion publique de services municipalisés (voirie, déchets, loisirs, sécurité publique, etc.) et non municipalisés (emploi, scolaire, social, jeunesse, etc.). En outre, différentes mesures de soutien au développement économique, social et culturel s'ancrent à cette échelle dite de proximité des acteurs.

À degrés divers, selon les territoires, ces quatre missions sont alimentées par une approche globale de la gouvernance. Chaque milieu MRC progresse en ce sens selon sa propre trajectoire communautaire. La globalité du visionnement collectif est généralement nourrie par un système de relations intersectorielles capables de concilier les municipalités, notamment les pôles urbains plus imposants. Ce qui occasionne l'allègement des replis corporatistes, la circulation de l'information, la vigie sur les nouveaux enjeux territoriaux, le ciblage d'intérêts supérieurs ainsi que la priorisation de choix collectifs pour des dossiers communautaires à réaliser souvent en partenariats. À travers la grande diversité d'arrangements institutionnels sur les 101 territoires MRC, notre analyse des faits propose trois grandes catégories de gouvernance que voici :

- 20 à 25 territoires largement ruraux illustrent une très bonne dynamique organisationnelle facilitée par l'équivalence du pouvoir (taille base fiscale - lettres patentes - etc.) entre les municipalités. Treize de ces territoires possèdent un préfet élu au suffrage;

- 20 à 25 territoires largement urbains disposent d'un leadership organisationnel approprié de la principale municipalité fusionnée ou pas en 2001;

- 50 à 60 territoires de mixité urbaine - rurale souffrent d'une faible ou moyenne dynamique communautaire largement limitée par la nonéquivalence du pouvoir (taille - base fiscale lettres patentes - etc.) entre les municipalités.

Il est à souligner que parmi la moitié des territoires MRC globalement mieux gouvernés que les autres, une vingtaine de collectivités se démarquent clairement du lot en matière d'appropriation collective de leur devenir autour d'un projet global de communauté. Ces best practices montrent la voie du progrès communautaire pour les autres territoires.

En ce sens, signalons que les récentes mesures gouvernementales affectent de facto ce progrès en matière de gouvernance territoriale. Les coupures dans les ressources publiques éliminent en effet totalement la politique rurale avec son utile formule de pactes ruraux épousant l'échelle MRC. Aussi, les CLD sont affaiblis financièrement dans leur mission. Pourtant la capacité organisationnelle générale de ces territoires supralocaux mériterait davantage de soutien dans un esprit d'efficacité générale de la gouvernance publique. Trois défis nous apparaissent impératifs à relever à cet égard. 
En ce sens, signalons que les récentes mesures gouvernementales affectent $d e$ facto ce progrès en matière de gouvernance territoriale.

Le premier grand défi réside dans la relance de l'exercice d'aménagement à associer avec la planification du développement, incluant les modalités de gestion des choix retenus comme orientations et actions. Cette relance nécessitera une importante mise à jour de la LAU (loi sur l'aménagement et l'urbanisme) dans un contexte général de multiplication des normes environnementales. On sait que depuis le début des années 1980, l'exercice de confection du cadre d'aménagement de chaque MRC représente un processus collectif complexe au niveau technique et contraignant au niveau politique. En conséquence, la confection des schémas d'aménagement peine à compléter sa $2^{\mathrm{e}}$ génération pour l'ensemble des territoires MRC. Tandis que l'élaboration d'une $3^{\mathrm{e}}$ mouture de ce cadre fut lancée au sein de quelques territoires seulement, en dépit de l'impérative nécessité de ces outils devant en principe être renouvelés tous les cinq ans.

Le $2^{\mathrm{e}}$ grand défi réside dans la coordination intermunicipale. Puisque les fusions effraient les élus autant locaux que nationaux, la solution optimale pour des gains d'efficacité réside dans les mises en commun de ressources. À cet effet, la collaboration volontaire a beaucoup progressée au cours des dernières décennies sous la pression de finances locales. Mais elle fait face à la lourde

\section{CONCLUSION}

\section{On constate en substance que les cinq catégories de composantes territoriales du Québec contemporain sont utiles à différents niveaux pour la gouvernance publique.}

Cette courte analyse a permis d'illustrer des faits à propos de l'évolution de la réforme de la gouvernance territoriale au Québec qui fut amorcée dans les années 1960. Ce qui nous a permis de situer la période très actuelle dans son contexte évolutif plus large. L'exercice serait à contrainte de l'inégale répartition du pouvoir et des moyens fiscaux entre les municipalités de tailles et de richesses variables. Ce constat colle bien aux milieux ruraux, mais aussi aux zones périurbaines qui illustrent de multiples enjeux organisationnels. Les classiques ententes intermunicipales doivent ainsi innover vers de nouvelles formules partenariales pour lesquelles Québec pourrait s'impliquer non seulement avec des incitatifs financiers, mais aussi avec des accompagnements techniques. Accompagnements qui pourront aussi servir l'appropriation territoriale de nouvelles responsabilités « décentralisables », notamment dans les secteurs des ressources naturelles, du transport collectif et de l'environnement.

Le dernier grand défi des territoires MRC réside dans la collaboration entre les agences publiques indépendantes qui exercent à cette échelle dans l'emploi, la santé, les services sociaux, le soutien au développement, le scolaire, l'éducation supérieure, le transfert technologique, le transport, la sécurité publique, etc. Au sein des MRC urbaines du Québec, ce secteur public non municipal, fragmenté par fonctions distinctes, s'avère au total des dépenses généralement plus important que le secteur municipal. Si de nombreux partenariats existent déjà, le potentiel restant demeure élevé. Des synergies potentielles sont à portée de main dans un esprit d'actions nouvelles pour les collectivités territoriales. À cet effet, se pose clairement l'enjeu de la participation des agences fédérales présentes sur le terrain.

reprendre plus en détails ${ }^{22}$ tel un chapitre important dans l'histoire politico-administrative du Québec. Ce numéro spécial de la revue Organisations et territoires participe en ce sens à cette œuvre nécessaire. Nous y avons contribué par quelques jalons de base d'un constat général sur la gouvernance territoriale.

On constate en substance que les cinq catégories de composantes territoriales du Québec contemporain sont utiles à différents niveaux pour la gouvernance publique. Les municipalités locales possèdent leur raison d'être dans la gestion 
publique des biens et services collectifs de base. Les régions administratives ont illustré leur efficacité comme aires de gestion des programmes gouvernementaux. Les territoires supralocaux MRC exercent les missions d'aménagement, de développement et aussi de gestion publique de biens et services intermunicipaux et non municipaux qui nécessitent à la fois la proximité des clients et d'importants bassins de ceux-ci. Aussi, de nouvelles zones reliées à des problématiques territoriales particulières deviennent de plus en plus pertinentes pour l'intervention publique. En outre, finalement, de nombreux pôles de différentes tailles et vocations représentent des cibles incontournables pour l'intervention publique concernée par la structuration des territoires.

Alors que la majorité des projecteurs actuels sont ciblés sur l'enjeu fiscal, se posent à notre avis deux autres questions importantes. Elles s'inscrivent pour le Québec dans un contexte universel de gouvernance territoriale ${ }^{23}$. D'abord celle concernant l'imbrication globale à l'échelle du Québec de ces cinq types de composantes territoriales. À cet effet, rappelons que le modèle $\mathrm{du}$ « système urbain hiérarchisé » a joué un rôle fort utile jadis pour ordonnancer adéquatement les interventions publiques en matière d'aménagement, de gestion et de développement. En regard de la recomposition contemporaine des territoires, un nouveau modèle général d'organisation de l'espace Québec devient essentiel pour optimiser le positionnement de chaque territoire. L'autre question incontournable concerne les modalités institutionnelles de coordination interne des différents acteurs présents sur les territoires, soit les élus locaux, les représentants de l'État, la société civile et le secteur privé. À cet effet, les nombreuses pratiques de médiation territoriale expérimentées au cours des dernières décennies s'avèrent riches de leçons, notamment les best practices très actuelles. Les réponses appropriées à ces deux questions s'avèrent nécessaires pour que la prochaine étape de la réforme territoriale en cours au Québec soit autre que cosmétique.

\section{BIBLIOGRAPHIE ET NOTES}

${ }^{1}$ Voir les Cahiers du Conseil d'orientation économique du Québec ainsi que le Rapport de la Commis- sion provinciale d'urbanisme présidée par JeanClaude La Haye, Québec, Gouvernement du Québec, 1968.

${ }^{2}$ Voir le texte de Lionel Robert «L'espace et l'État», paru en 1978 dans la revue Critère, no 23, pp. 231-258.

${ }^{3}$ Voir l'ouvrage de Jean Meynaud et Jacques Léveillée (1973) « La régionalisation municipale au Québec, Montréal, Nouvelles Frontières.

${ }^{4}$ Voir le document de 1966 du ministère de l'Industrie et du Commerce intitulé « Division du Québec en dix régions administratives et 25 sous-régions administratives ", Bureau de recherches économiques, Québec, MIC.

${ }^{5}$ Voir les textes de Clermont Dugas (2002) «Une stratégie de renforcement des petits centres ruraux », Dans revue Organisations et territoires, vol. 11, no 1, pp. 11-16. Et aussi celui de Christel Alvergne (2008) "Les pôles d'excellence rurale ", dans revue Organisations et territoires, vol. 17, no 1, pp.73-78.

${ }^{6}$ Voir l'ouvrage collectif édité par A.J. Scott « Global City-Regions », New-York, Oxford University Press. ${ }^{7}$ Voir les chapitres 7 et 8 du livre «Territoires et développement : la richesse du Québec », publié en 2011 par Marc-Urbain Proulx aux Presses de l'Université du Québec.

${ }^{8}$ Voir le Rapport de la Commission d'étude sur les municipalités sous la présidence de Jacques Parizeau, Montréal, Union des municipalités du Québec, 1986.

${ }^{9}$ Avec un taux de participation de seulement $47 \%$ au scrutin municipal de 2013, 55\% des 8050 postes électifs furent comblés sans opposition, avec un minime $8 \%$ d'élus âgés entre 18 à 34 ans.

${ }^{10}$ Voir l'excellent texte de Robert Dugas intitulé «Bilan et analyse de la régionymie dans l'administration publique québécoise », Communication présentée au $1^{\text {er }}$ Congrès international sur la toponymie française en Amérique du Nord, Québec, 1984. Voir aussi le texte de Louis-Marie Bouchard « La régionalisation de l'administration au Québec », dans la revue Critère, no 24, Hiver 1979, pp. 35-40.

${ }^{11}$ Voir note no 7.

${ }^{12}$ Voir Jean-Jacques Simard (1979), « La longue marche des technocrates », Laval, Éditions Saint-Martin.

${ }^{13}$ Voir l'article de Marc-Urbain Proulx intitulé «Quarante ans de planification territoriale au Québec », paru dans M. Gauthier, M. Gariépy et M.O. Trépanier (dir), Renouveler l'aménagement et l'urbanisme, Montréal, Presses de l'Université de Montréal, 2008.

${ }^{14}$ Voir le document d'Yves Cartier et Marie Le Rouzès qui en 1987 analysait les résultats de la première génération de Conférences socio-économiques régionales comme procédure d'animation en multiples étapes de mobilisation et de montage de la faisabilité de projets.

${ }^{15}$ Voir le texte référé à la note no 9. 
${ }^{16}$ Voir l'article de Isabel Brochu et Marc-Urbain Proulx «La construction institutionnelle régionale » paru en 1995 dans la Revue d'administration publique du Canada, vol. 36, no 4, pp. 94-111.

${ }^{17}$ Voir le texte Diane-Gabrielle Tremblay « Réseaux locaux et districts industriels ", dans l'ouvrage collectif de M.U. Proulx 1994 « Développement économique », Québec, Éditions de la Fondation de l'entrepreneurship.

${ }^{18}$ Effets de débordement associés aux nouvelles entreprises générées par les économies de proximité (faibles couts de transaction; mobilités des talents; complémentarités de production; complicités professionnelles; circulation d'information; coopération; etc.) au sein des grandes zones économiques spécialisées des pays fortement industrialisés ou en industrialisation rapide.

${ }^{19}$ Voir le texte de Marc-Urbain Proulx intitulé «Nouveau cycle économique en périphérie nordique », paru en 2014 dans la revue Actualité Économique, vol. 90, no 2, pp. 121-144.

${ }^{20}$ Malgré l'expertise considérable à l'interne du gouvernement, Québec a néanmoins formé en 2003 une Commission d'étude sur la gestion de la forêt publique québécoise afin de faire davantage de lumière.

${ }^{21}$ Voir le texte de Marc-Urbain Proulx intitulé «Territoires de gestion et territoires d'émergence », dans l'ouvrage collectif de Guy Massicotte (2008) Sciences du territoire, Québec, Presses de l'Université du Québec.

${ }^{22}$ Voir trois petits ouvrages intéressants : Dossier spécial «Organisation des territoires » par MarcUrbain Proulx publié en 1998 par la revue Action Nationale; Vincent Lemieux (1998) « La décentralisation ", Québec éditons de l'IQRC; Richard Morin (2006) «La régionalisation au Québec», Montréal, Éditions Saint-Martin.

${ }^{23}$ Voir Graham Haughton et ali (2010) « The New Spatial Planning », London, Routledge; Simin Davoudi and Ian Strange (edit) (2009) « Conceptions of Space and Place in Strategic Spatial Planning, London, Routledge. 